\title{
Comparative Assessment of Orthogonal and Nonorthogonal Multiplexing Techniques for Differentiated Satellite Broadcasting Services
}

\author{
Farbod Kayhan*, Guido Montorsi ${ }^{\dagger}$, Giorgio Taricco ${ }^{\dagger}$ and Nader Alagha ${ }^{\ddagger}$ \\ ${ }^{*}$ Interdisciplinary Centre for Security, Reliability and Trust (SnT), \\ University of Luxembourg, 4, rue Alphonse Weicker, L-2721 Luxembourg \\ Email: farbod.kayhan@uni.lu \\ ${ }^{\dagger}$ Politecnico di Torino, DET, 10129, Toirno, Italy \\ Emails: guido.montorsi,giorgio.taricco@polito.it \\ ${ }^{\ddagger}$ European Space Technology Centre, ESA/ESTEC, Keplerlaan 1, 2200 AG Noordwijk ZH \\ Email: nader.alagha@esa.int
}

\begin{abstract}
This paper presents a comparative assessment of two multiplexing techniques for providing differentiated classes of services over a realistic broadcasting satellite channel under variable link conditions. A distinctive characteristic of satellite broadcasting channels is the non-linear characteristics of high power amplifier on-board of the satellite. In this scenario, the conventional additive white Gaussian noise (AWGN) channel with average power limitation is no longer an accurate model and nonlinear characteristics have to be taken into account. Orthogonal multiplexing (time sharing) techniques combined with variable coding and modulation are compared with hierarchical modulations with the goal of maximizing the number of broadcast channels over a given transponder while maintaining a target service availability. Hierarchical modulations are shown to provide better performance than orthogonal time division multiplexing (time-sharing) schemes for the AWGN channel. Here we design a practical transmitter scheme based on the hierarchical modulation and maximize the throughput by optimizing the achievable mutual information for finite size constellations. This will provide a lower bound on throughput which can be achieved by non orthogonal multiplexing scheme. We compare the performance of hierarchical modulation (non-orthogonal multiplexing) and time sharing techniques (orthogonal multiplexing) supporting two different service quality and service availability requirements for broadcasting the same content.
\end{abstract}

Index Terms-Satellite Broadcast Channel, Hierarchical Modulation, Digital Video Broadcasting via Satellite.

\section{INTRODUCTION}

The capability of satellites to rapidly setup services and provide multitude of Television (TV) channels at relatively low cost to a large number of subscribers has created a significant growth in the digital broadcasting satellite market in the past two decades. The quality of broadcasting video streams has evolved from standard definition quality to high-definition. More bandwidth demanding video contents such as UltraHigh Definition (UHD) and 3D-TV create new opportunities for direct broadcasting satellite, particularly targeting home large screens. Direct satellite broadcasting to end-users (known as direct to home, DTH services) faces some technical challenges. One major challenge of maintaining service availability over satellite channel is the atmospheric fading, particularly when operating at higher frequency bands such as $\mathrm{Ku}$ or $\mathrm{Ka}-$ band.

In conventional satellite broadcasting systems, the service availability is guaranteed by dimensioning the end-to-end system taking into account the fading conditions. This is achieved by enforcing a higher link margin. Since the signal power flux density observed by the receiver is limited (due to regulatory issues or system coordination), the increase of the link margin would require a larger receiver antenna gain (i.e. bigger dish size) or a more protected modulation and coding scheme with a lower decoding threshold. The use of more protected modulation and coding would reduce the spectral efficiency, that can be linked to the number of broadcast TV channels per satellite transponder.

More sophisticated technical solutions have been designed based on variable coding and modulation (VCM), simulcasting the same content with different quality, and scalable video coding (SVC) that can provide differentiated quality of service and graceful degradation of the broadcast content [1]. These techniques allow for adjusting transmission robustness according to the service availability targets, and to differentiate services according to the quality of service requirements.

The use of VCM in conjunction with simulcast can also be used to guarantee service continuity in the presence of a heavy atmospheric fading, while at the same time offering a high quality service in the absence of rain attenuation. By allowing a tolerable degradation in the picture quality during a heavy fading event, it is possible to significantly increase the overall system spectral efficiency. The use of variable modulation and coding for different layers of information content represent time sharing or time division multiplex with no time overlapping hence orthogonal transmission.

In this paper we compare time sharing approach with alternative multiplexing solutions where the transmission of different layers overlap in time (super-imposed), hence it is non-orthogonal transmission. 
The achievable rate of the linear broadcast channels has been studied by Cover [2]. The author shows that by superimposing the high rate and low rate information one can achieve rates superior to that of the orthogonal time-sharing scheme. In particular, for degraded AWGN broadcast channel, the superposition provides the maximum achievable rate [2]. Several strategies have been introduced in the literature to implement the superimposed information in a practical system. Among these techniques, Superposition modulation (SM), Hierarchical modulation (HM) and multilevel coding (MLC) have received particular attention in the literature [3]-[6].

In [7], the authors propose a hierarchical 16-APSK modulation scheme to improve the performance of satellite broadcast channels for some selected code rates. Simulations over a realistic channel show a gain of maximum $10 \%$ in available rate with respect to that of the orthogonal scheme. Vargas and et. al. [8] study the effect of satellite system impairments on multilevel coding systems. In particular, they show that in the multilevel coding scheme, the first level is not affected by the satellite channel impairments while the last level degrades slightly with respect to BICM scheme. However, a measure to compare these two schemes is not provided.

A distinctive characteristic of the satellite broadcasting channels is the operation close to the saturation of the onboard high power amplifier (HPA). This allows to gain the maximum efficiency of the on-board power amplifiers. In such scenarios, the conventional additive white Gaussian noise (AWGN) channel with average power constraint over the signal space is no longer an accurate model. In order to have a more realistic analysis of the satellite broadcast channel, we need to take into account also the nonlinear characteristics of the channel caused by the HPA.

The main question is whether strategies employed over the AWGN channel can outperform the orthogonal scheme also in the presence of nonlinearity. The theoretical results are not available for non-orthogonal multiplexing over the nonlinear channels. However, several experimental studies are available in the literature, see for example [9] and [10].

In this paper we design a practical transmitter scheme based on the hierarchical modulation and maximize the throughput by optimizing the achievable mutual information of the finite size constellations. This will provide a lower bound on throughput which can be achieved by non-orthogonal multiplexing scheme. We show that this lower bound is larger than the time sharing orthogonal multiplexing achievable rate if the attenuation difference between users accessing the two services is large. For optimizing the finite size constellations we use the simulated annealing algorithm which has been shown to be very effective. For details on optimization algorithm we kindly refer the readers to [11] and the references within.

The rest of this paper is organized as follows. In Section II we describe the system model and the basic concepts. We review the theoretical results for the linear AWGN channel in section III. In section IV we study the nonlinear channel and compare the orthogonal multiplexing with the hierarchical

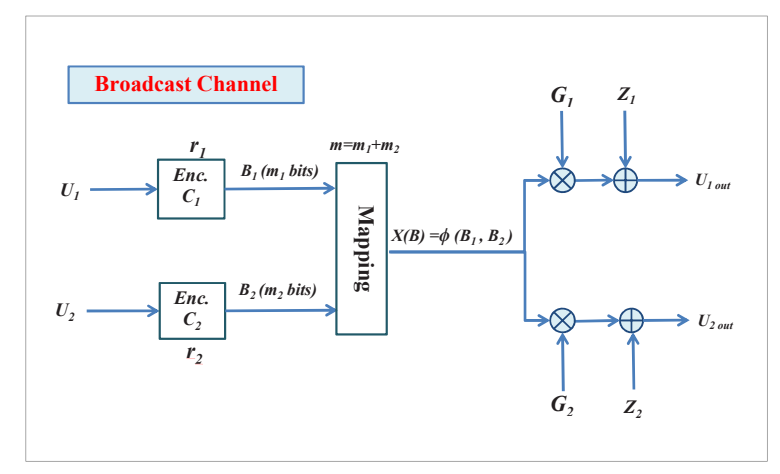

Fig. 1. Broadcast channel, the hierarchical transmission.

modulation scheme. Finally, we conclude the paper in Section $\mathrm{V}$ and suggest some further studies.

\section{System Model}

In this paper we focus on the transmission of $K$ services over a satellite communication link. The symbol generated by the encoder of each service is represented by $B_{i} \in \mathcal{U}_{i}$ for $i=1, \ldots, K$ where $\mathcal{U}_{i}$ denotes the symbol alphabet. The transmitted signal is represented by the complex mapping

$$
X=\phi\left(B_{1}, \ldots, B_{K}\right) .
$$

For the sake of notation simplicity, we restrict our attention to the case of $K=2$ broadcasting services in most parts of this paper, so that $X=\phi\left(B_{1}, B_{2}\right)$.

The communication link for a particular user of the system is affected by a random but constant gain $G$ and by additive Gaussian noise $Z$. As a result, the equivalent channel model (with compensated attenuation) is expressed as follows

$$
Y_{i}=X+G_{i}^{-1} Z_{i}=\phi\left(B_{1}, B_{2}\right)+G_{i}^{-1} Z_{i}, \quad i=1,2 .
$$

Here we assume that $Z \sim \mathcal{N}_{c}\left(0, P_{Z}\right)$, so that $G^{-1} Z \sim$ $\mathcal{N}_{c}\left(0, \alpha P_{Z}\right)$, where we define $\alpha \triangleq|G|^{-2}$ as the channel (power) attenuation, whose cumulative distribution function (CDF) is given by $F_{\alpha}(x)=P(\alpha<x)$. The main parameter which characterizes the satellite channel is the average signal to noise ratio (SNR) defined as $\Gamma \triangleq \mathbb{E}\left[|X|^{2}\right] / P_{Z}$. In Fig. 1 we present a general block diagram of the broadcasting channel. The set of two services are first encoded with code rates $r_{i}$, generating the sequence of coded bits $c_{i}$ that are mapped into the constellation points by the function $\phi$. Note that the encoded bits are grouped in blocks of $m_{i}$ bits, to form the symbol which we have denoted by $B_{i}$. The constellation cardinality is then $M=2^{m_{1}+m_{2}}$. A practical design of the transmitter scheme requires to carefully select the binary codes, the modulation efficiencies for each service, the complex constellation set and the labeling function $\phi\left(B_{1}, B_{2}\right)$.

We assume that $U_{1}$ represents the low-rate data-stream, less demanding in terms of bit rate, more demanding in terms of 
availability. Its required bit rate, measured in bits per channel use, is $R_{1}$ and the availability is $P_{a 1}=F_{\alpha}\left(\alpha_{1}\right)$. On the other hand, $U_{2}$ represents the high-rate data-stream, more demanding in terms of bit rate, less demanding in terms of availability. Its required bit rate is $R_{2}$ and the availability is $P_{a 2}=F_{\alpha}\left(\alpha_{2}\right)$. Therefore, we assume in the rest of this paper that $P_{a 1}>P_{a 2}$ or equivalently $\alpha_{1}>\alpha_{2}$.

\section{A. Availability}

The random nature of the channel attenuation $\alpha$ has a major implication on the system performance. In fact, a communication service is said to be available whenever the attenuation is lower than a certain threshold $\alpha^{*}$. Thus, we call service availability the probability $P\left(\alpha<\alpha^{*}\right)$.

\section{B. Channel Efficiency}

In order to define the channel efficiency we first introduce the relative rate vector $\left(1, \rho \triangleq R_{2} / R_{1}\right)$. Given the broadcast channel parameters, our goal is to find the modulation set (image of $\phi$ ), the optimal allocation of the modulation bits to each service and the labeling map such that the mutual information vector $\left(I_{1}, I_{2}\right)$ satisfies the inequality

$$
\left(I_{1}, I_{2}\right) \succeq \beta\left(1, R_{2} / R_{1}\right)
$$

with $\beta$ as large as possible. We call $\beta$ the channel efficiency of the broadcast system. The information vector in (2) is defined as

$$
\begin{aligned}
& I_{1}=I\left(Y_{1} ; B_{1}\right) \\
& I_{2}=I\left(Y_{2} ; B_{2} \mid B_{1}\right)
\end{aligned}
$$

The $\beta$ can then be defined as

$$
\beta=\min \left(I\left(Y_{1} ; B_{1}\right), \frac{R_{1}}{R_{2}} I\left(Y_{2} ; B_{2} \mid B_{1}\right)\right)
$$

In a system adopting the BICM approach, the pragmatic mutual information $I^{(p)}$ should be substituted to $I$ :

$$
\begin{aligned}
I_{1}^{(p)} & =\sum_{k=1}^{m_{1}} I\left(Y_{1} ; B_{1}^{k}\right) \\
I_{2}^{(p)} & =\sum_{k=1}^{m_{2}} I\left(Y_{2} ; B_{2}^{k} \mid B_{1}\right),
\end{aligned}
$$

where $B_{i}^{k}$ denotes the $k$-th bit of the binary label associated to $i$-th service.

The channel efficiency can be defined in the similar way as in (4) by substituting the mutual information with the pragmatic mutual information. The required code rates $r_{1}$ and $r_{2}$ are then obtained as

$$
\left(r_{1}, r_{2}\right)=\left(I_{1} / m_{1}, I_{2} / m_{2}\right) .
$$

Notice that $\beta$ should be optimized under the given power constraint on the channel. We will review briefly the high power amplifier model and the possible power constraints in the next subsection.

\section{Predistortion and Hard-Limiter function}

In the following we use a hard-limiter instead of the real AM/AM amplification curves. The generality of this approach can be justified as follows. Consider an arbitrary nonlinear amplifier function $g(A)$, monotonic for $A \in\left(0, A_{\max }\right)$, with $g\left(A_{\max }\right)=A_{\max }$. Then, define the predistortion function:

$$
p(A)= \begin{cases}g^{-1}(A) & A \in\left(0, A_{\max }\right] \\ A_{\max } & A \in\left(A_{\max }, \infty\right)\end{cases}
$$

This predistortion function satisfies the property:

$$
g(p(A))= \begin{cases}A & A \in\left(0, A_{\max }\right] \\ A_{\max } & A \in\left(A_{\max }, \infty\right)\end{cases}
$$

Therefore, the application of the predistortion function to the power amplifier input transforms its response into

$$
\hat{g}(A) \triangleq g(p(A))=\min \left(A, A_{\max }\right) .
$$

This guarantees the generality of considering the hard-limiter instead of the real nonlinear amplification curve as far as concerns the AM-AM characteristics. Thus, in the following we assume that the nonlinear amplifier considered operates according to the hard-limiter model, i.e., under an amplitude constraint $|X| \leq A_{\max }$. In principle, we are interested in the case that the HPA is acting very near to its saturation point. In this case, the parameter that characterize the satellite communication system is the peak SNR defined as $\Gamma_{\max } \triangleq A_{\max }^{2} / P_{Z}$. where the average power constraint over the signal space is substituted by the maximum power constrain.

\section{LINEAR CHANNEL}

In this section we evaluate the channel efficiency $\beta$ corresponding to the orthogonal and non-orthogonal multiplexing schemes. Focusing on the two-service broadcast satellite system we assume that the symbols $U_{1}$ and $U_{2}$ represent the two data streams to be transmitted.

For each case our goal is to optimize the input distribution, depending on the rate and availability constraints, in order to maximize the channel efficiency $\beta$.

\section{A. Orthogonal scheme}

In this paper we consider the time division multiplexing (TDM) scheme for orthogonal multiplexing. In Fig. 2 a block diagram of the transmitter for orthogonal scheme is shown. The optimization in this case is rather simple [12]. Let us set

$$
\gamma_{i} \triangleq \frac{\Gamma}{\alpha_{i}}, \quad i=1,2
$$

so that $\gamma_{1}<\gamma_{2}$. Given the SNR $\Gamma$ and the attenuation thresholds $\alpha_{1}, \alpha_{2}$ deriving from the availability constraints, we know that the mutual information per channel use associated to each service is given by:

$$
C_{i}=\log \left(1+\gamma_{i}\right) \quad \text { bits per channel use. }
$$

The channel efficiency $\beta_{\mathrm{ORTH}}$ in this case is maximized adopting a sharing of resource proportional to the ratio between the required service rate $R_{i}$ to the mutual information of the channel: 


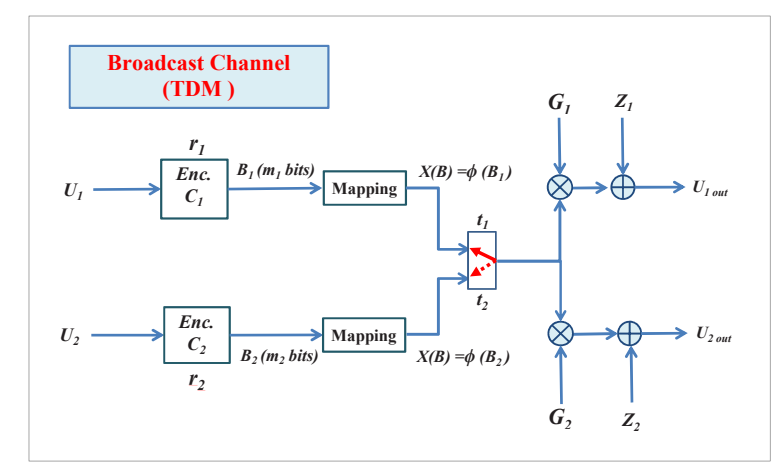

Fig. 2. Orthogonal broadcasting scheme: time division multiplexing.

$$
I_{i}=t_{i} C_{i} \rightarrow t_{i}=\frac{R_{i} / C_{i}}{R_{1} / C_{1}+R_{2} / C_{2}} .
$$

The resulting channel efficiency can be obtained as

$$
\beta_{\mathrm{ORTH}}=\frac{R_{1}}{R_{1} / C_{1}+R_{2} / C_{2}} .
$$

\section{B. Nonorthogonal scheme}

In this case the optimization of the input distribution is a little more complex and requires the optimization of the mapping function $\phi\left(B_{1}, B_{2}\right)$.

This mapping function, in its simplest form, can be reduced to

$$
\phi\left(B_{1}, B_{2}\right)=\phi_{1}\left(B_{1}\right)+\phi_{2}\left(B_{2}\right) .
$$

This corresponds to the standard superposition modulation (SM) approach, which is shown to achieve the capacity for linear broadcast systems [12], [3], [4].

The channel efficiency in this case is given by:

$$
\begin{aligned}
\beta_{\mathrm{HM}}=\max _{\substack{\xi_{1}, \xi_{2}>0 \\
\xi_{1}+\xi_{2}=1}} \min \{ & \log _{2}\left(1+\frac{\xi_{1} \gamma_{1}}{1+\xi_{2} \gamma_{1}}\right), \\
& \left.\frac{R_{1}}{R_{2}} \log _{2}\left(1+\xi_{2} \gamma_{2}\right)\right\} .
\end{aligned}
$$

Since the two arguments of the minimum above are, respectively, monotonically increasing and monotonically decreasing with $\xi_{1}$, the maximum is attained when they are equal, namely:

$$
\beta_{\mathrm{HM}}=\log _{2}\left(1+\frac{\xi_{1}^{*} \gamma_{1}}{1+\xi_{2}^{*} \gamma_{1}}\right)=\frac{R_{1}}{R_{2}} \log _{2}\left(1+\xi_{2}^{*} \gamma_{2}\right)
$$

for some $\xi_{1}^{*}, \xi_{2}^{*} \in(0,1)$ such that $\xi_{1}^{*}+\xi_{2}^{*}=1$. It is worth noting that $\xi_{1}$ and $\xi_{2}$ determine the fraction of power allocated to the transmission of the symbols $B_{1}$ and $B_{2}$, respectively. More precisely, the SNR allocated to the symbols are $\xi_{1} \Gamma$ and $\xi_{2} \Gamma$, respectively. Taking into account the threshold attenuations $\alpha_{1}$ and $\alpha_{2}$ we get the mutual information expressions in eq. (9), where it is assumed that $B_{1}$ is decoded first.
A natural question arising is how much larger is the $\beta_{\mathrm{HM}}$ with respect to the $\beta_{\mathrm{ORTH}}$. As far as we know, no upper bound has been found in the literature. We can prove the following upper bound for $\beta_{\mathrm{HM}}$.

Theorem III.1. Assuming an average power limit and the notations defined previously in this paper, if $R_{1}<R_{2}$ and $P_{a 1}>P_{a 2}$ (hence, $\alpha_{1}>\alpha_{2}$ ), then for every $\Gamma, \Gamma_{I}, R_{1}, R_{2}$, the following inequalities hold:

$$
\beta_{\mathrm{ORTH}}<\beta_{\mathrm{HM}}<2 \beta_{\mathrm{ORTH}} .
$$

Proof.

$$
\begin{aligned}
\frac{\beta_{\mathrm{HM}}}{\beta_{\mathrm{ORTH}}}= & \beta_{\mathrm{HM}} \cdot\left(\frac{1}{\log _{2}\left(1+\gamma_{1}\right)}+\frac{R_{2} / R_{1}}{\log _{2}\left(1+\gamma_{2}\right)}\right) \\
= & \frac{1}{\log _{2}\left(1+\gamma_{1}\right)} \log _{2}\left(1+\frac{\xi_{1}^{*} \gamma_{1}}{1+\xi_{2}^{*} \gamma_{1}}\right) \\
& +\frac{1}{\log _{2}\left(1+\gamma_{2}\right)} \log _{2}\left(1+\xi_{2}^{*} \gamma_{2}\right) \\
= & 1-\frac{\log _{2}\left(1+\xi_{2}^{*} \gamma_{1}\right)}{\log _{2}\left(1+\gamma_{1}\right)}+\frac{\log _{2}\left(1+\xi_{2}^{*} \gamma_{2}\right)}{\log _{2}\left(1+\gamma_{2}\right)} \\
\geq & 1,
\end{aligned}
$$

since $\gamma_{1}<\gamma_{2}$ and $\log _{2}(1+\xi \gamma) / \log _{2}(1+\gamma)$ is an increasing function of $\gamma$ for $\gamma>0$ and for any given fixed $\xi \in(0,1)$.

Moreover, since $\xi<\log _{2}(1+\xi \gamma) / \log _{2}(1+\gamma)<1$ for all $\gamma>0$ and $\xi \in(0,1)$, we can see that

$$
\frac{\beta_{\mathrm{HM}}}{\beta_{\mathrm{ORTH}}} \leq 2-\xi_{2}^{*}<2
$$

which concludes the proof of the theorem.

\section{NONLINEAR CHANNEL}

\section{A. Orthogonal Scheme}

Optimizing the value of $\beta$ is again simple for the orthogonal system. Given the peak SNR, $\Gamma_{\max }$, and the attenuation thresholds $\alpha_{1}, \alpha_{2}$ deriving from the availability constraints, the capacity is achieved by the distribution proposed by SmithShamai-Bar-David (SSB) (see [13] and [14]), leading to:

$$
C_{i, \text { peak }}=\mathcal{C}_{\text {peak }}\left(\frac{\Gamma_{\max }}{\alpha_{i}}\right)
$$

Similar to the linear channel, the $\beta_{\mathrm{ORTH}}$ is given as:

$$
\beta_{\mathrm{ORTH}}=\frac{R_{1}}{R_{1} / C_{1, \text { peak }}+R_{2} / C_{2, \text { peak }}} .
$$

Notice that we use the same notation for $\beta$ in both linear and non-linear channel.

\section{B. Non-Orthogonal Multiplexing and Constellation Optimiza- tion}

Assuming the superposition scheme $\phi\left(B_{1}, B_{2}\right)=\phi_{1}\left(B_{1}\right)+$ $\phi_{2}\left(B_{2}\right)$ usually leads to a optimization procedure which is less complicated than the general case. However, our preliminary results showed that the performance achievable by superposition modulation is not always the best over the assumed 


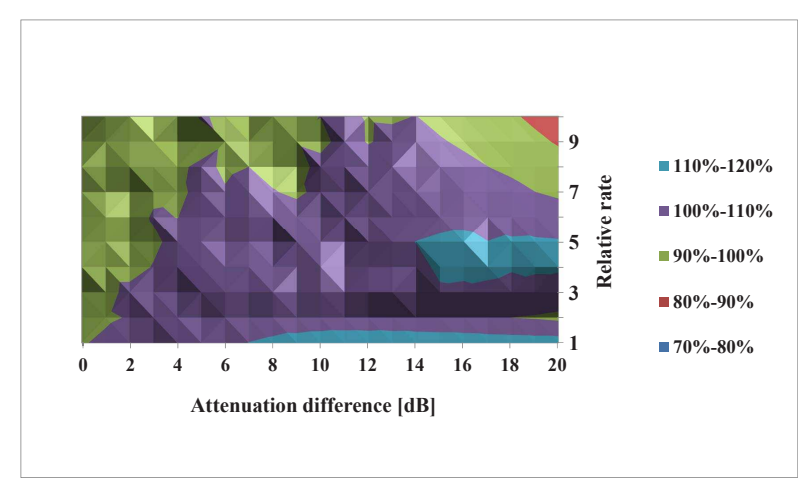

Fig. 3. HM gain with respect to the orthogonal scheme capacity as a function of attenuation differences and relative rates for pragmatic receiver under the peak power constraint.

nonlinear channel, therefore, we decided to resort to the more general approach based on the mapping function $\phi\left(B_{1}, B_{2}\right)$.

Nevertheless, the general optimization problem seems to be too difficult to tackle. In this paper, we confine ourselves to a sub-optimal approach consisting in the restriction of the possible values of $\phi\left(U_{1}, U_{2}\right)$ to a finite cardinality set of size equal to an integer power of two. Our goal is then to optimize the finite constellation set and compare it with the orthogonal scheme capacity for given broadcast channel parameters. For optimizing the finite size constellations we use the simulated annealing algorithm which has been shown to be very effective. For details on optimization algorithm we kindly refer the readers to [11] and the references within.

The achievable mutual information of a finite constellations provides only a lower bound for the capacity of the HM scheme. We confine ourselves to constellations with 16 points. In Fig. 3 we present the gain $\beta_{\mathrm{HM}}^{\text {opt }} / \beta_{\mathrm{ORTH}}$ of the hierarchical modulation with respect to the orthogonal scheme capacity. We have calculated the reference $\beta_{\text {ORTH }}$ using the Shamai and Bar-David results [14]. As it can be observed in Fig 3, the gain is larger than one for some range of attenuation difference $\left(\alpha_{1} / \alpha_{2}\right.$ in $\left.\mathrm{dB}\right)$ and relative rate $\rho$ values, indicating that the HM can indeed provide better system performance with respect to the orthogonal scheme also over the non-linear broadcast channel. For example, the purple region labeled by $100 \%-110 \%$ indicates 0 to 10 percent gain by using HM scheme. On the other hand, the green region labeled by by $90 \%-100 \%$ indicates 0 to 10 percent loss with respect to the orthogonal multiplexing. In particular, it can be observed that the gain increases as the attenuation difference become larger. This is usually the case when operating at high frequency bands (for example Ka band), or when the attenuation distribution is really steep (for example in tropical climates).

\section{CONCLUSIONS AND FURTHER STUDIES}

In this paper we compared the orthogonal and nonorthogonal multiplexing for a satellite broadcasting system with on-board non-linear high power amplifier. Our objective function is a properly defined channel efficiency for such channels. We first review the theoretical results for linear channel showing that non-orthogonal multiplexing has a larger channel efficiency than the orthogonal (TDMA) scheme. We further prove that the channel efficiency for the non-orthogonal system can not exceed twice that of the orthogonal scheme. For the peak-power limited channels, the theoretical results for maximum channel efficiency exists only for the orthogonal systems. We designed a practical system by optimizing the finite size constellations and showed that the channel efficiency of such a system is larger than that of the orthogonal limit for large attenuation differences values between the services. In other words, we provide a lower bound for the non-orthogonal system performance which exceeds the orthogonal scheme limits for some set of parameters.

In order to have a fair comparison between two systems, it would be interesting to calculate the performance of orthogonal system using finite sized constellations. Research in this direction is ongoing.

\section{ACKNOWLEDGMENT}

This work is funded by the European Space Agency, ESA-ESTEC, Noordwijk, The Netherlands, under contract no. 4000109715. Opinions, interpretations, recommendations and conclusions expressed herein are those of the authors and are not necessarily endorsed by the European Space Agency.

\section{REFERENCES}

[1] Nader S. Alagha and Pantelis-Daniel Arapoglou,"Technology Trends for Ka-Band Broadcasting Satellite Systems", 7th EAI International Conference on Wireless and Satellite Systems (WiSATS 2015), Bradford, July 2015.

[2] T. M. Cover, "Broadcast Channels", IEEE Trans. Inform. Theory, vol.IT-18, No. 1. Jan. 1972.

[3] H. Imai and S. Hirakawa A New Multilevel Coding Method Using Error Correcting Codes, IEEE Transactions on Information Theory, 23:371377, May 1977

[4] J. Huber et. al,, Coded Modulation by Multilevel-Codes, Overview and State of the art, Shaker Verlag, Aachen, Germany, 1998.

[5] E. Chen, et. al., Layered Modulation Concept for Digital Satellite Broadcast Transmissions DIRECTV, 31 May 2002, DVB-TMS2-0006

[6] Hong Jiang et. al, Providing Local Content in a Hybrid Single Frequency Network Using Hierarchical Modulation, IEEE Trans. On Broadcasting, Dec 2010.

[7] H. Meric, J. Lacan, F. Arnal, G. Lesthievent and M.-L Boucheret,"Combining Adaptive Coding and Modulation With Hierarchical Modulation in Satcom System", IEEE Transactions on Broadcasting, vol. 59, no. 4, pp. 627-637, Dec. 2013.

[8] A. Vargas, C. Keip, W. Gerstacker, and M. Breiling,"Effect of Satellite System Impairments on a Multilevel Coding System for Satellite Broadcasting", In Proceedings of the Vehicular Technology Conference (VTC Spring11), Budapest, Hungary, May 2011.

[9] Hugo Meric et. al, Trade-off between spectrum efficiency and link unavailability for hierarchical modulation in DVB-S2 systems, Vehicular Technology Conference (VTC 2013 - Spring), 2 June 2013 - 5 June 2013 (Dresden, Germany).

[10] S.Wang, et. al. "On Enhancing Hierarchical Modulation”, IEEE International Symposium Broadband Multimedia Systems and Broadcasting, 2008.

[11] F. Kayhan and G. Montorsi, "Constellation design for memoryless phase noise channels",IEEE Transactions on Wireless Communications, vol.13, no.5, pp.2874,2883, May 2014.

[12] T. M. Cover,"Some Advances in Broadcast Channels" Technical Report September 17, 1974, Stanford University. 
[13] J.G. Smith, "The information capacity of amplitude- and varianceconstrained scalar Gaussian channels," Inform. Contr., vol. 18, pp. 203219,1971 . See also, "On the information capacity of peak and average power constrained Gaussian channels," Ph.D. dissertation, Dep. Elec. Eng., Univ. of California, Berkeley, CA, Dec. 1969.

[14] S. Shamai (Shitz) and I. Bar-David, "The capacity of average and peak-power-limited quadrature Gaussian channels," IEEE Trans. Inform. Theory, vol. 41, pp. 1061-1071, July 1995. 